\title{
Color of Interference and Joint Encoding and Medium Access in Large Wireless Networks
}

\author{
Nithin Sugavanam, C. Emre Koksal, Atilla Eryilmaz \\ Department of Electrical and Computer Engineering \\ The Ohio State University \\ Columbus, Ohio 43201
}

\begin{abstract}
We address the problem of resource allocation in extended wireless networks in the presence of user dynamics. We first characterized the first two order statistics of the interference in such networks where users arrive according to a spatiotemporal Poisson process where each user has a certain amount of information to transmit, specified by a probability distribution. We show that, user dynamics and single user behavior dominate the auto-covariance function. We then formulated an optimization problem in order to develop a joint power and rate allocation scheme for systems in which users transmit simultaneously up on arrival, without any collision avoidance or scheduling. We then provide an iterative algorithm to solve the optimization problem, thereby obtaining the rate and power allocation strategy, taking into consideration the correlation of interference and the energy constraints. We also observe that our scheme leads to an interference averaging phenomenon as the transmission duration becomes large, which reduces the outages in the packet transmissions. Our scheme is a low overhead and completely asynchronous over users.
\end{abstract}

\section{INTRODUCTION AND MOTIVATION}

Conventionally, resource allocation and Medium Access Control(MAC) algorithms assume a separation of timescales. Timescale separation implies that the flow-level/user dynamics (user arrival and departure) occur at a much larger timescale than the time required for the scheduling algorithms to optimally allocate resources such as transmission power, encoding rates, and routes to the users with respect to a performance measure like throughput, spectral efficiency or delay. With the time scale separation, associated schemes assume ergodicity of the parameters such as the service rates and queue sizes, making the design simpler [1].

However, in many cases, user dynamics vary in smaller time scales, e.g., in networks where the arriving users, transmit very small amount of information and depart. In the presence of such time scales, algorithms designed with the time-scale separation suffer from a significant performance loss from overhead, especially due to the communication of control messages. Also, without ergodicity of queue sizes, most algorithms fail to provide provable guarantees. For example, MaxWeight may not achieve throughput optimal resource allocation in presence of flow level dynamics as shown in [2]. Also, dynamic interference management schemes, which handle both power allocation and scheduling at the physical layer, require synchronization and information sharing, and are not suited for large networks with short-lived flows. This motivates us to consider the use of asynchronous MAC schemes, in which user dynamics are handled in the physical layer.

To that end, we consider a model for an extended wireless network, in which nodes arrive at the network according to a spatio-temporal Poisson process to transmit a certain amount of packets, distributed according to some distribution and leave after they are done. The main difference between our scheme and the standard collision avoidance or scheduling schemes is that, we assume nodes transmit simultaneously one the same channel, except for those which are within a certain neighborhood of an active receiver. Thus, a receiver experiences a superposition of signals originating from all the users, attenuated by a certain amount of path loss, increasing with the distance from the receiver. Consequently, the amount of interference observed by a receiver at a given time $t$ is a correlated random process. Using tools from queueing theory, we derive the autocovariance function of the observed interference power in Section III-A. Similar studies on temporal modeling of the interference power can be found in [3] and [4]. Then, we state the problem as an optimal transmission power and encoding rate allocation, subject to an average energy constraint. Each user has a certain maximum amount of average energy that they consume; thus, our objective here is to maximize the bits/joule/user. This metric is directly tied to the area spectral efficiency [5] of the system, as we discuss in Section II-D. Based on the statement, we develop an asynchronous, joint transmission power and encoding rate allocation policy in Section II-C, whereby each arriving user selects a power and encoding rate over time in response to their observed interference at the time of their arrival.

\section{System Model and Problem Statement}

\section{A. User Dynamics}

We consider a random extended multi-cellular uplink network as shown in Fig. 1. Arriving users are distributed according to a spatio-temporal Poisson process with intensity $\lambda$ users $/ \mathrm{s} / \mathrm{m}^{2}$. Each user remains active for a random duration of time, $T \mathrm{~s}$ with distribution $F_{T}(t)$. We assume that there is no collision avoidance or scheduling.

\section{B. Channel and Interference Model}

We define distance $d_{0}$ from the origin called the reuse distance, within which only a single transmitter can be active. The signal power observed by the receiver at origin, originating 


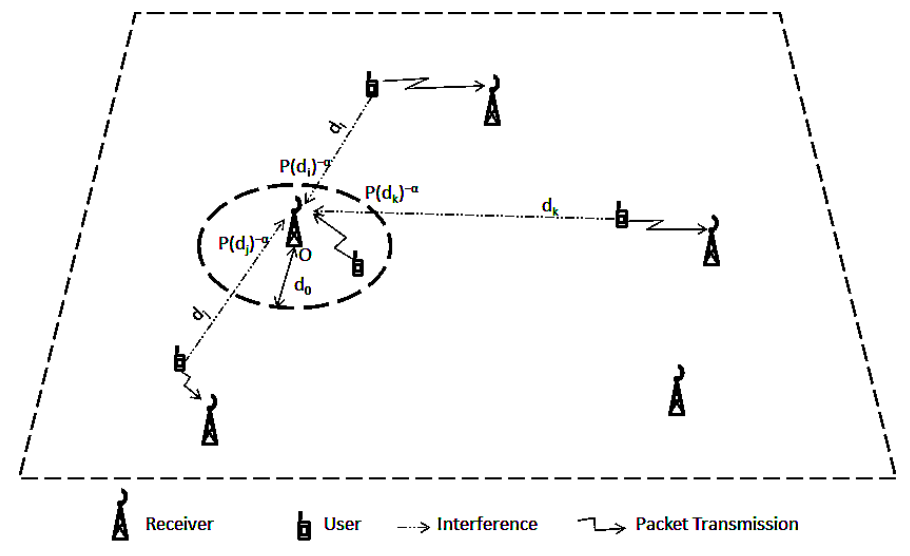

Fig. 1. Multicellular Up-link wireless network

from a user transmitting at Power, $P$, located at a distance $d_{i}>d_{0}$ is $P\left(d_{i}\right)^{-\alpha}$ where $\alpha>2$ is the path-loss exponent. The noise is additive Gaussian noise with bandwidth $W$ and noise power spectral density as $N_{0}$. We assume that each receiver treats interference as Gaussian noise.

\section{Transmission Strategy}

Each user arriving at time $t$ observes the interference power level, $Z(t)$, and chooses a transmission power $P_{Z(t)}$ and an encoding rate $R_{Z(t)}$ to transmit $T$ packets in succession. In this paper, we assume each packet contains a separate independent message, encoded using a block code of rate $R_{Z(t)}$. If, during the transmission of any packet, the instantaneous SINR exceeds $2^{R_{Z(t)}}-1$, that packet cannot be decoded correctly and an outage occurs. The overall cumulative information rate for a user is identical to $T R_{Z(t)}$ times the fraction of packets that do not experience an outage ${ }^{1}$ Our joint power and rate strategy takes into consideration the expected energy constraint, $E$ over the transmission duration and maximizes the expected accumulated mutual information over all packets that do not experience an outage. Our strategy utilizes the temporal correlation in the interference, which in turn is governed by the user level dynamics.

\section{Problem Statement}

The definition of the area spectral efficiency for a system with an instantaneous SINR identical to $\gamma(t)$ is given as

$$
C_{a}=\frac{1}{\pi d_{0}^{2}} \mathrm{E}[\log (1+\gamma(t))],
$$

where the expectation is over the received power level and the interference power. Area spectral efficiency is basically identical to the achievable spectral efficiency, normalized with the area of frequecy reuse. One can realize that, as the area increases, the denominator on $C_{a}$ increases, but the numerator (spectral efficiency) increases, since the expected interference decreases. Area spectral efficiency is of direct relevance to

\footnotetext{
${ }^{1}$ Note that, if there were a single message to be encoded over $T$ packets, then we would need to use an outer code at a rate lower than $R_{Z(t)}$, to make for the outages experienced by a few of the packets.
}

us, since $C_{a} / \lambda$ bits/Hz/user gives us an upper bound on the amount of information communicated per user per unit bandwidth. In our system described in Section II-C, the power level remains constant at $P_{Z(t)}$ over a duration $T$ and the area spectral efficiency can be written as

$$
\begin{gathered}
\frac{1}{\pi d_{0}^{2}} \int_{Z(t)} f_{Z(t)}(z(t)) \int_{t}^{t+T}\left[\int_{Z(\tau)} f_{Z(\tau) \mid Z(t)}(z(\tau) \mid z(t))\right. \\
\left.\log _{2}\left\{1+\frac{P_{Z(t)}}{N_{0} W+Z(\tau)}\right\} d z(\tau)\right] d \tau d z(t)
\end{gathered}
$$

for any given $d_{0}$, where $f_{Z(t)}(z(t))$ and $f_{Z(\tau) \mid Z(t)}(z(\tau) \mid z(t))$ denote the marginal distribution and the conditional distribution of interference level respectively for a given frequency reuse radius $d_{0}$. Note that, the area spectral efficiency is not achievable subject to a finite delay (transmission duration), $T$. As per our packetized transmission strategy, described in Section II-C, the arriving user transmits information encoded over $T$ packets (each of which is transmitted over unit time) at rate $R_{Z(t)}$ chosen at time $t$ and decoded individually. If we use the accumulated mutual information, instead of the spectral efficiency on the numerator, we obtain:

$$
\begin{gathered}
\frac{1}{\pi d_{0}^{2}} \int_{Z(t)} f_{Z(t)}(z(t)) \sum_{i=1}^{T} R_{z(t)} \\
\mathrm{P}\left[\log _{2}\left(1+\frac{P_{Z(t)}}{N_{0} W+Z(t+i)}\right) \geq R_{Z(t)} \mid z(t)\right] d z(t),
\end{gathered}
$$

which is basically $R_{Z(t)}$ times the sum of probabilities that a packet, encoded at rate $R_{Z(t)}$ and transmitted at time $t+i$ with a power level $P_{Z(t)}$, is decoded successfully.

We formulate the optimal power and rate allocation problem as follows:

$$
\begin{aligned}
& \max _{P_{Z(t)}, R_{Z(t)}} \int_{Z(t)} f_{Z(t)}(z(t)) \sum_{i=1}^{T} R_{z(t)} \\
& \quad \operatorname{Pr}\left[\log _{2}\left\{1+\frac{P_{Z(t)}}{N_{0} W+Z(t+i)}\right\} \geq R_{Z(t)} \mid z(t)\right] d z(t) \\
& \text { subject to } \int_{Z(t)} P_{Z(t)} f_{Z(t)}(z(t)) d z(t) \leq \frac{E}{T}
\end{aligned}
$$

where the objective function in (4) is the expected accumulated information by a user over $T$ packets and (5) enforces the constraint that cumulative energy consumed over the entire transmission duration cannot exceed $E$. Note that we got rid of the area $\pi d_{0}^{2}$ in the denominator, as we fix $d_{0}$ before the transmission. The packet outage probability can be written in terms of the conditional pdf of the interference as:

$$
\begin{aligned}
& \mathbf{P}\left[\log _{2}\left(1+\frac{P_{Z(t)}}{N_{0} W+Z(t+i)}\right) \geq R_{Z(t)} \mid z(t)\right]
\end{aligned}
$$

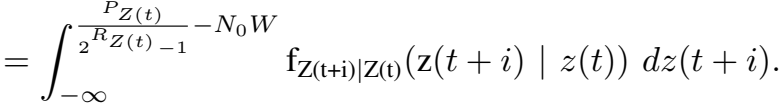




\section{Performance Analysis and Design}

In order to solve the optimization problem in (4), we need to understand the statistics of the temporal variations of the interference power, $Z(t)$. To that end, the primary objective of this section is to derive the auto-covariance function of $Z(t)$ and subsequently provide a solution to the optimization problem in (4), to obtain a strategy for joint power and rate allocation.

\section{A. Color of Interference}

Theorem 1: Let the auto-covariance function, the variance, and the mean of $Z(t)$ be denoted with $K_{Z}(\tau), \sigma_{Z}^{2}$, and $\mu_{Z}$, respectively. Then,

$$
\begin{aligned}
K_{Z}(\tau) & =\frac{\pi \lambda P^{2}}{\alpha-1} d_{0}^{2-2 \alpha} \int_{|\tau|}^{\infty}[1-F(s)] d s, \\
\mu_{Z} & =\frac{2 \pi \rho P}{\alpha-2} d_{0}^{2-\alpha}, \\
\sigma_{Z}^{2} & =\frac{\pi \rho P^{2}}{\alpha-1} d_{0}^{2-2 \alpha},
\end{aligned}
$$

where $\rho=\lambda \mathrm{E}[T]$ users $/ \mathrm{m}^{2}$ is the utilization factor (per unit area) for the network.

Proof: The proof is provided in [6].

There are some remarkable observations one can make from Theorem 1. Firstly, the spatial and the temporal components of $K_{Z}(\tau)$ are separate and they take effect in the form of a product. Furthermore, the shape of $K_{Z}(\tau)$ is dictated by the service distribution of a single user. For instance, in Fig. 2, we illustrate $K_{Z}(\tau)$ for a deterministic $T$, which is identical to 5 with probability 1 . This dominance of single user service duration is somewhat surprising, since, as the network size extends, the number of simultaneous interferers approaches $\infty$. One might think that the total interference becomes whiter and whiter, as the number of interferers increase, however this does not turn out to be true. The user arrival rate, transmission power, and the frequency reuse region size affect the scale of the covariance function, but not the shape of it. In a sense, the temporal behavior of the interference is the same regardless of whether there is a single interferer or many interferers. Finally, it is also worth mentioning that, if $T$ is heavy tailed, then the interference process becomes long range dependent.

\section{B. Joint Power and Rate Allocation}

To solve the constrained optimization problem $(4,5)$ we evaluate the Lagrangian by using (6) as follows:

$$
\begin{aligned}
& \mathcal{L}\left(\lambda, P_{z(t)}, R_{z(t)}\right)=\int_{Z(t)} \sum_{i=1}^{T}\left[R_{z(t)}\right. \\
& \int_{-\infty}^{\frac{P_{Z(t)}}{2^{R} Z(t)}-1}-N_{0} W \\
& -\lambda\left(P_{Z(t)}-\frac{E}{T}\right) f_{Z(t)}(z(t)) d z(t) .
\end{aligned}
$$

Note that, due to the non-convexity of the problem, the solution via the Lagrangian does not provide optimality. However,

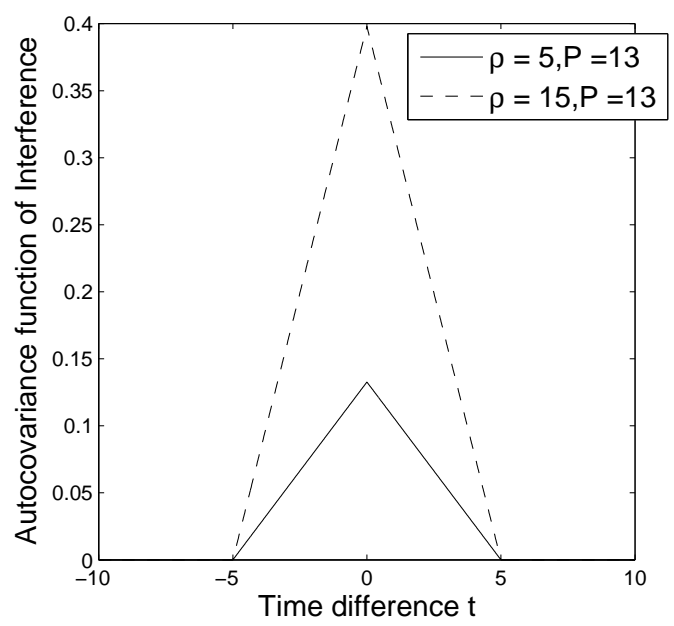

Fig. 2. Auto-covariance function of the interference level observed at origin with $\lambda=1$ and 3 users $/ \mathrm{sec} / \mathrm{m}^{2}$ and $\mathrm{P}=13 \mathrm{~W}, \alpha=5$, and $T=5 \mathrm{sec}$.

by exploiting the special structure of the parameters, we evaluate the optimal solution. For that purpose, we make use of an iterative framework. We note that transmission power $P_{z(t)}$ is constrained, while encoding rate $R_{z(t)}$ has no boundaries. We successively find the solution with respect to $R_{z(t)}$, while keeping $P_{z(t)}$ constant and find the solution with respect to $P_{z(t)}$, while keeping $R_{z(t)}$ constant until a convergence criterion is met. Given energy constraint $\mathrm{E}$ and number of packets $\mathrm{T}$, we propose the following algorithm :

1) Initialize $P_{Z(t)}^{(0)}=E / T \forall Z(t), \lambda=\lambda_{0}$, and $\mathrm{i}=1$.

2) Using (10), we solve $\forall Z(t)$ :

$$
\begin{aligned}
R_{Z(t)}^{(i)} & =\underset{R_{Z(t)}}{\arg \max } \mathcal{L}\left(\lambda, P_{Z(t)}^{(i-1)}, R_{Z(t)}\right) . \\
\lambda & =\underset{\bar{\lambda}}{\arg \min } \max _{P_{Z(t)}} \mathcal{L}\left(\bar{\lambda}, P_{Z(t)}, R_{Z(t)}^{(i)}\right) . \\
P_{Z(t)}^{(i)} & =\underset{P_{Z(t)}}{\arg \max } \mathcal{L}\left(\lambda, P_{Z(t)}, R_{Z(t)}^{(i)}\right)
\end{aligned}
$$

3) If $\left\|P_{Z(t)}^{(i)}-P_{Z(t)}^{(i-1)}\right\|>\epsilon_{1}$ or $\left\|R_{Z(t)}^{(i)}-R_{Z(t)}^{(i-1)}\right\|>\epsilon_{2}$, where $\epsilon_{1}, \epsilon_{2} \ll 1$ then increment $\mathrm{i}$ by 1 , and repeat step 2

\section{NUMERICAL INVESTIGATIONS AND OBSERVATIONS}

In this section we investigate the impact of various system and design parameters on the performance of our joint power and rate allocation strategy developed in Section III-B. In particular, we first study the effect of user-activity duration $T$ on the resulting power and rate allocation over time, and the corresponding throughput performance. Also, we study the behavior with expanding bandwidth which is important to understand under ultra-bandwidth communication possibilities. Throughput this section, we assume that the interference power level $Z(t)$ is a Gaussian process, characterized by the first two order statistics provided in Theorem 1. Thus, 


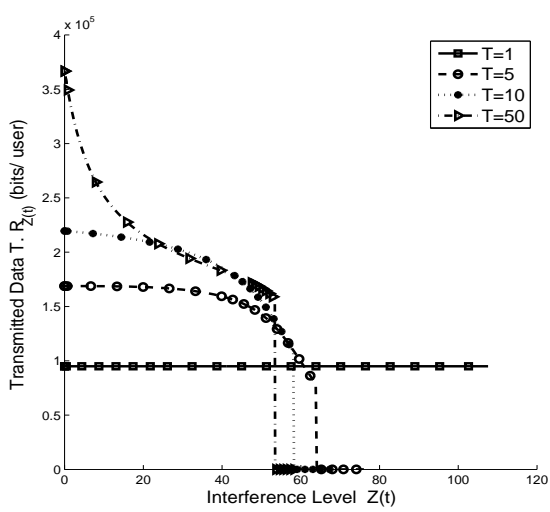

(a) Rate Allocation Strategy

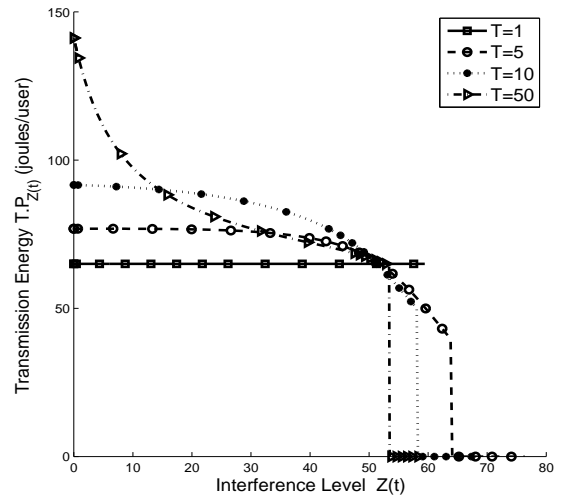

(b) Power Allocation Strategy

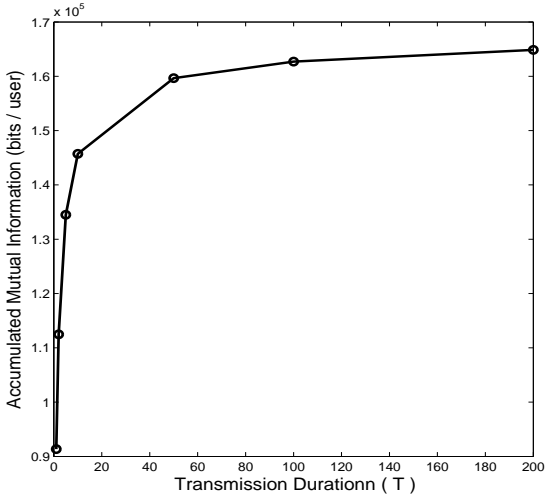

(c) Relationship between Accumulated Mutual Information and Number of packets $\mathrm{T}$

Fig. 3. Influence of $\mathrm{T}$ on the Rate and Power allocation Strategies

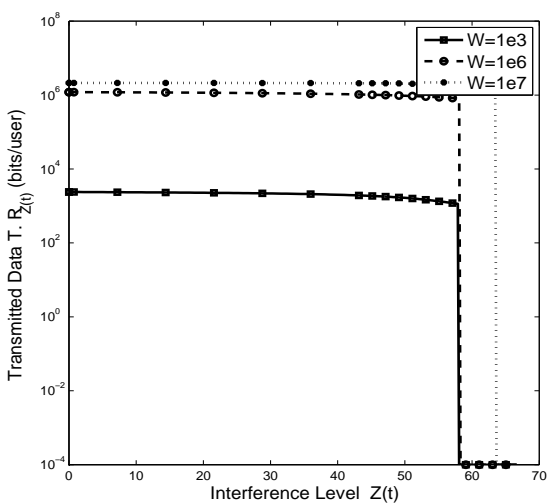

(a) Rate Allocation Strategy

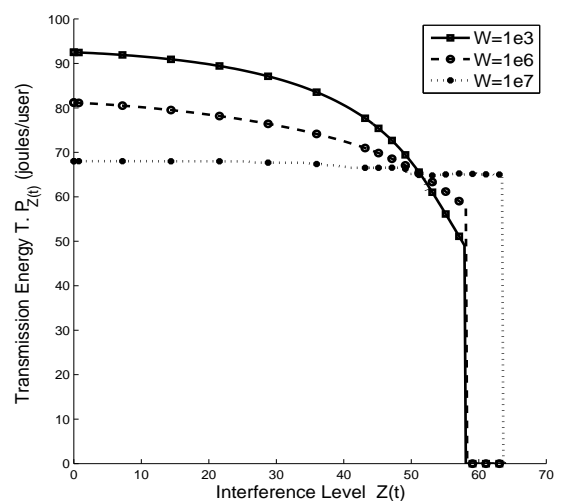

(b) Power Allocation Strategy

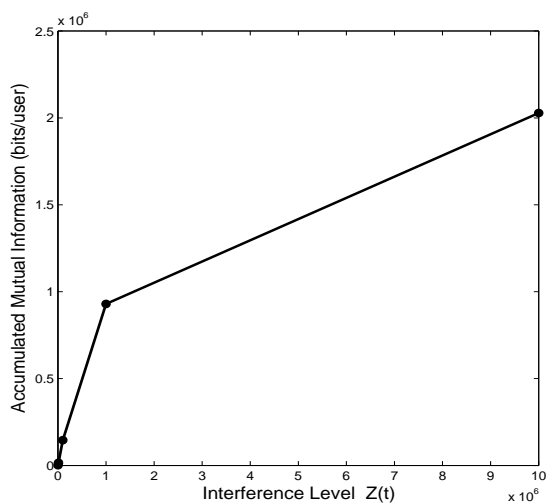

(c) Relationship between Accumulated Mutual Information and Number of packets, $\mathrm{T}$

Fig. 4. Influence of Channel Bandwidth on the Power and Rate allocation strategies

$f_{Z(t)}(z(t)) \sim \mathcal{N}\left(\mu_{Z}, \sigma_{Z}^{2}\right)$ and $f_{Z(t+i) \mid Z(t)}(z(t+i) \mid z(t)) \sim$ $\mathcal{N}\left(\mu_{Z}+\frac{K_{Z}(\tau)\left(Z(\tau)-\mu_{Z}\right)}{K_{Z}(0)}, \sigma_{Z}^{2}-\frac{K_{Z}(\tau)^{2}}{K_{Z}(0)}\right)$.

\section{A. Effects of $T$ on Joint Allocation and Performance:}

We recall that the power allocation of each incoming user over its entire transmission duration $T$ is subject to an average energy constraint of $E$, which is fixed. Upon arrival, each user will observe the interference level at its intended receiver to determine its power and rate allocation over its transmission duration according to our policy described in Section III-B. Since the temporal correlation of interference is also a function of $T$, as derived in Lemma 1 , it is of interest to see how the allocation should respond to $T$ and whether longer $T$ improves performance.

Figure 3 reveals the typical nature of our allocations and throughput performance with varying $T$, illustrated for the parameters of $\alpha=4, d_{0}=2 \mathrm{~m}, \lambda=1$ users $/ \mathrm{s} / \mathrm{m}^{2}, W=10 \mathrm{kHz}$. First, not surprisingly we observe that when $T=1$, the power and rate allocations are insensitive to the observed interference level $Z(t)$ at time $t$, because the temporal correlation between the observation and the time of transmission is zero. However, as $T$ increases the operation of the algorithm possesses dual behavior: above a threshold level, no transmission takes place; and below it both the power and rate allocations exhibit a waterfilling-type allocation, transmitting at a higher rate with higher power when the observed interference is lower. The resulting throughput performance in Figure 3(c) reveals that, by adapting its power and rate to the colored interference that $T$ generates, the algorithm achieves increasing levels of throughput with larger $T$.

\section{B. Effects of $W$ on Power and Rate Allocation:}

In this investigation, we study the effect of the channel bandwidth $W$ on the nature and the performance of our joint algorithm. Such a scenario is of interest in view of the possibility of utilizing ultra-bandwidth communication strategies in the implementation of our solution. We note that as $W$ increases, the effect of interference becomes relatively smaller when compared to the channel noise, thereby operating the system under a noise-dominant regime. In Figure 4, we illustrate the rate/power allocation and the resulting performance of our policy for varying values of $W$, where we use the same parameters as in the previous investigation with $T=5$.

Our results reveal that our joint allocation converges to 
a power and rate profile, whereby a static threshold on interference emerges above which no transmission is made, and below which a constant rate allocation and non-uniform power allocation is selected. The rate allocation is insensitive to the interference level, whereas its magnitude decreases with $W$. The latter phenomenon is expected since large $W$ implies larger noise power. On the other hand, the power allocation is more adaptive to interference, assigning larger powers for lower values of interference.

\section{CONCLUSION}

In this paper, we first characterized the first two order statistics of the interference in an extended wireless network with user dynamics. Our results indicate that user dynamics and single user behavior dominate the auto-covariance function. We formulated an optimization problem in order to develop a joint power and rate allocation scheme for systems in which users transmit simultaneously up on arrival, without any collision avoidance or scheduling. We then provide an iterative algorithm to solve the optimization problem, thereby obtaining the rate and power allocation strategy, taking into consideration the correlation of interference and the energy constraints. We also observe that our scheme leads to an interference averaging phenomenon as the transmission duration becomes large, which reduces the outages in the packet transmissions.

Our scheme is a low overhead, asynchronous MAC scheme that can be applied to energy limited and green communication networks, cognitive networks, and direct-sequence CDMA in ad-hoc network setting.

\section{REFERENCES}

[1] A. Proutiere, Y. Yi, T. Lan, and M. Chiang, "Resource allocation over network dynamics without timescale separation," in Proceedings of the 29 th conference on Information communications, ser. INFOCOM'10. IEEE Press, 2010, pp. 406-410.

[2] P. M. van de Ven, S. C. Borst, and S. Shneer, "Instability of maxweight scheduling algorithms." in INFOCOM. IEEE, 2009, pp. 1701-1709.

[3] X. Yang and A. Petropulu, "Co-channel interference modeling and analysis in a poisson field of interferers in wireless communications," Trans. Sig. Proc., vol. 51, no. 1, pp. 64-76, Jan. 2003.

[4] K. Gulati, B. L. Evans, and S. Srikanteswara, "Joint temporal statistics of interference in decentralized wireless networks." Trans. Sig. Proc., vol. 60, no. 12 , pp. 6713-6718, 2012, to appear.

[5] M.-S. Alouini and A. J. Goldsmith, "Area spectral efficiency of cellular mobile radio systems," IEEE Trans. Veh. Technol, vol. 48, pp. 1047-1066, 1999.

[6] N. Sugavanam, E. Koksal, and A. Eryilmaz, "Color of interference and joint encoding and medium access in large wireless networks," Tech. Rep., 2012. 